\title{
TRASPLANTE AUTÓLOGO "POTENCIADO" DE CÉLULAS MADRE CD34+ DE MÉDULA ÓSEA, EN PACIENTES CON ANGINA REFRACTARIA. ESTUDIO PILOTO.
}

\author{
MARGARITA INES BLANCOํ, HENRY OLIVEROS ${ }^{2}$, BENJAMÍN OSPINO ${ }^{3}$, \\ OSCAR ÁLVAREZZ ${ }^{4}$ JUAN FELIPE VÁSQUEZ ${ }^{5}$, JENNIFFER P. ÁVILA MSc ${ }^{6}$ \\ LUZ MABEL ÁVILA-PORTILLO $c P h D^{7}$
}

${ }^{1}$ Cardióloga, Servicio de Cardiología, Hospital Militar Central, ${ }^{2}$ Epidemiólogo, Profesor Asociado Universidad de la Sabana, Hospital Militar Central,

${ }^{3}$ Hematólogo, Stem Medicina Regenerativa,

${ }^{4}$ Rehabilitador Cardiaco, Hospital Militar Central,

${ }^{5}$ Médico, Egresado de la UMNG,

${ }^{6}$ Ciencias Biológicas, Stem Medicina Regenerativa,

${ }^{7}$ Ciencias Farmacéuticas, Hospital Militar Central, Stem Medicina Regenerativa.

Correspondencia: Margarita Inés Blanco Guarín

margaritaines_blanco@hotmail.com.Tel.3168780484-3486868

Recibido: Agosto 10 de 2015

Aceptado: Diciembre 17 de 2015

\section{Resumen}

Objetivo: Evaluar la eficacia a 6 meses del trasplante autólogo de células madre $\mathrm{CD}_{34}^{+}$de médula ósea "potenciado", mediante pre-condicionamiento isquémico en pacientes con angina refractaria.

Métodos: Estudio piloto con 14 pacientes con angina refractaria, clase funcional clínica mayor o igual a III (NYHA y CCS), del Servicio de Cardiología del Hospital Militar Central. Fueron seleccionados por conveniencia 14 pacientes los cuales se asignaron aleatoriamente a 2 grupos, el primero (intervención) con trasplante autólogo de células madre CD34+ de médula ósea potenciado mediante pre-condicionamiento isquémico por vía intravenosa más tratamiento médico convencional, y el segundo (control) con tratamiento médico convencional. Se realizaron mediciones basales a 6 meses del umbral de angina/isquemia medido en mets y clase funcional.

Resultados: Al comparar las medianas, el cambio en el valor umbral de angina/isquemia 6 meses después, para el grupo intervenido fue de 3.5 mets vs 0.9 mets, para el grupo control $\mathrm{P}=0.013$. No se registraron complicaciones inherentes al tratamiento.

Conclusiones: En esta investigación, los pacientes con angina refractaria intervenidos con trasplante autólogo de células madre de médula ósea $\mathrm{CD} 34^{+}$potenciado mediante pre-condicionamiento isquémico mostraron mejoría del umbral de angina y clase funcional a 6 meses.

Palabras clave: Células madre hematopoyéticas, Angina de pecho, Precondicionamiento isquémico. 


\title{
TRANSPLANT AUTOLOGOUS “ENHANCED” STEM CELLS CD34+ BONE MARROW IN PATIENTS WITH REFRACTORY ANGINA \\ PILOT STUDY
}

\begin{abstract}
Objective: To evaluate the efficacy of 6 months of autologous CD34+ stem cells in bone marrow "enhanced" by ischemic preconditioning in patients with refractory angina.

Methods: A pilot study with 14 patients with refractory angina, with a functional class greater than or equal to III clinical (NYHA and CCS), of the Department of Cardiology of the Central Military Hospital. They were selected by convenience 14 patients who were randomly assigned to two groups, the first (intervention) with autologous stem cells $\mathrm{CD} 34^{+}$bone marrow powered by ischemic preconditioning, intravenously more conventional medical treatment, and the second (control) with conventional medical treatment. Baseline measurements were performed and six months' threshold angina/ischemia measured in mets and functional class.
\end{abstract}

Results: Comparing the medians, the change in threshold value angina/ischemia six months later for the intervention group was 3.5 mets vs 0.9 mets for the control group $\mathrm{P}=0.013$. No inherent complications to treatment were recorded.

Conclusions: In this study patients with refractory angina who underwent autologous stem cell transplant of bone marrow $\mathrm{CD}_{34}{ }^{+}$powered by ischemic preconditioning, showed an improvement in threshold angina and functional class of 6 months.

Keywords: Hematopoietic stem cells, Angina pectoris, Ischemic preconditioning.

\section{TRANSPLANTE AUTÓLOGO “MELHORADO” STEM CELLS MEDULA ÓSSEA CD34+ EM PACIENTES COM ANGINA REFRATÁRIA: ESTUDO PILOTO}

Resumo

Objetivo: Avaliar a eficácia de 6 meses de células-tronco autólogas CD34 + na medula óssea "aumentada" pelo pré-condicionamento isquêmico em pacientes com angina refratária.

Métodos: Um estudo piloto com 14 pacientes com angina refratária, com classe funcional maior ou igual a III clínica (NYHA e CCS), Do Departamento de Cardiologia do Hospital Militar Central. Eles foram selecionados por conveniência 14 pacientes que foram aleatoriamente designados para dois grupos, a primeira (intervenção) com células-tronco autólogas $\mathrm{CD} 34^{+}$medula óssea alimentado por pré-condicionamento isquêmico, tratamento intravenoso mais convencional, e o segundo (controle) com tratamento médico convencional. Medidas de linha de base foram realizadas e seis meses de angina limiar /isquemia medido em mets e classe funcional.

Resultados: Comparando as mediana, a alteração do valor limiar angina / isquemia seis meses depois para o grupo de intervenção foi de 3,5 mets vs 0,9 mets para o grupo de controlo $P=0,013$. Não foram registadas complicações inerentes ao tratamento.

Conclusões: Neste estudo, pacientes com angina refratária submetidos a transplante de células-tronco autólogas de medula óssea $\mathrm{CD}_{3} 4^{+}$alimentado por pré-condicionamento isquêmico, apresentaram melhora da angina limiar e classe funcional de 6 meses.

Palavras-chave: células-tronco hematopoéticas, Angina de peito, Pré-condicionamento isquêmico 


\section{Introducción}

La angina refractaria es una entidad frecuente que afecta más del $9 \%$ de los pacientes con enfermedad coronaria significativa, los cuales no son candidatos a revascularización coronaria quirúrgica o percutánea $(1,2)$. El progreso de medidas terapéuticas, especialmente revascularización coronaria, ha llevado a disminución de mortalidad, pero con alta probabilidad de persistencia de síntomas $(3,4)$. La angina refractaria es un problema complejo y creciente, que corresponde a pacientes en quienes los mecanismos primarios de compensación ya han sido activados al máximo durante los episodios de isquemia crónica y son insuficientes, por lo cual continúan con angina y limitación en su calidad de vida a pesar de haber recibido tratamiento convencional óptimo de acuerdo a las guías para el manejo de enfermedad cardiaca isquémica estable (Farmacológico, prevención, rehabilitación, revascularización quirúrgica o percutánea) $(5,6)$, siendo considerados "Sin opción de tratamiento" (7), el problema principal de estos pacientes es la calidad de vida más que la mortalidad $(8,9)$, esta última ha sido reportada en $3.9 \%$ al año, $14 \%$ a los 5 años y $28 \%$ a los 9 años, cifra inferior a la reportada anteriormente $(10,11)$, consistente con estudios posteriores (12) y comparable con la registrada después de revascularización coronaria (13), por esto, los esfuerzos están dirigidos primordialmente a mejorar la calidad de vida. Teniendo en cuenta que en estos pacientes han agotado las posibilidades terapéuticas y que la angina está mediada principalmente por hipoperfusión coronaria, donde la restauración de la micro circulación es especialmente importante (14), surge como opción de tratamiento complementario el trasplante de células madre, buscando neo vascularización especialmente mediante angiogénesis, procedimiento con un amplio potencial, pero todavía en fase de investigación.

El uso de células madre para regeneración vascular fue preconizado en 1.997 por Asahara (15), quien publica el aislamiento de células progenitoras endoteliales circulantes de médula ósea con capacidad angiogénica. El trasplante de células madre en corazón humano se inició en la década pasada, la primera publicación fue hecha por Menasché P (16) en Francia en el 2001, su uso en regeneración cardiovascular se basa en estudios pre clínicos, clínicos, meta análisis y revisiones sistemáticas $(10,17-28)$, con resultados que reportan que el trasplante de células madre puede conducir al aumento de micro circulación y perfusión miocárdica a pesar de la oclusión permanente de vasos epicárdicos, con aumen- to de la densidad capilar, mejoría de clase funcional, umbral de angina, frecuencia de angina y calidad de vida; sin embargo, en ellos se encuentran variaciones en muchos parámetros como tipo y número de células madre utilizadas, uso o no de factor estimulante de colonias de granulocitos (G-CSF), vías de administración y parámetros de evaluación, entre otros. Aunque los resultados muestran tendencia al beneficio, algunos son sub-óptimos, discrepantes o dispersos, falta definir la correlación con los parámetros analizados y los mecanismos mediadores, de tal forma que quedan preguntas por resolver que requieren estudios pre clínicos y clínicos con suficiente poder estadístico, uno de cuales se encuentra en curso (29).

Dentro de las preguntas más importantes por definir están: tipo de trasplante, tipo y número de células madre, uso o no de G-CSF, vía de administración, retención, transdiferenciación, mecanismos de acción y mecanismos de potenciación. Aunque no hay una respuesta definitiva para ninguna de las preguntas las consideraciones son las siguientes:

Tipo y número de células madre: las más utilizadas en enfermedad cardiaca isquémica han sido provenientes de médula ósea, se han usado varios tipos, aisladas o mixtas, las que han mostrado hasta este momento mayor potencial angiogénico y las más utilizadas son las células mononucleares hematopoyéticas (HSC) $\mathrm{CD}^{2} 4^{+}(17)$, y recientemente ha tomado fuerza el uso de células progenitoras endoteliales (CPE) (30). En cuanto al número de células madre, hay reportes preclínicos y clínicos en diferentes sentidos $(10,31)$, el beneficio no está necesariamente relacionado con un mayor número de células madre administradas (10). El número de células debe ser regulado teniendo en cuenta, en primer lugar, que entre las células madre trasplantadas y el tejido receptor pueden producirse diferentes respuestas, entre ellas competencia y antagonismo (32) y, en segundo lugar, porque el mecanismo de acción indirecto puede ser más importante que el directo.

Vía de administración: Se han utilizado vías intracoronaria, intramiocárdica y venosa ya sea retrógrada por seno coronario o periférica. La vía intracoronaria requiere vaso permeable, lo cual no siempre es posible en pacientes con angina refractaria, la vía más usada ha sido la intramiocárdica que es la más directa pero tiene problemas relacionados con un rápido barrido, baja retención y no está exenta de riesgos (10), la vía venosa ha sido la menos usada, el estudio experimental de Freyman (33) muestra que por esta vía la mayoría de las células madre trasplantadas son atra- 
padas en otros órganos pero las células madre utilizadas fueron mesenquimales que son células grandes fácilmente retenidas. Como se planteó anteriormente, las que tienen mayor potencial de neovascularización son las células madre mononucleares hematopoyéticas que son más pequeñas y tienen un tránsito cerca de 30 veces mayor que las mesenquimales (34). El estudio de Hou (35), con células mononucleares de Medula Ósea en el que se comparan 3 vías de administración muestra que la retención en el miocardio por todas las vías es muy baja, la mayoría son atrapadas especialmente por pulmón en todas las modalidades, y aunque la mayor retención se hace por vía Intramiocárdica, los resultados son menos consistentes que con las otras vías y no hay diferencias significativas entre esta vía y la venosa retrógrada.

Retención, diferenciación y posibles mecanismos de acción: El trasplante de células madre en enfermedades cardiacas ha mostrado que la retención y diferenciación es muy baja con cualquier metodología (36-39). La retención en las primeras 24 horas es menor del 10\%, en la primera semana desaparecen el $90 \%$ de las células retenidas y al mes la retención es menor del 1\%, además de la retención muy baja, experimentalmente se ha encontrado que solo 2-3\% presentan fenotipo endotelial o de cardio miocito y sin embargo, los resultados son positivos (15), lo que permite plantear la importancia del efecto indirecto (40).

Potenciación: Uno de los factores que puede estar afectando el resultado del trasplante de células madre es la pobre señalización del miocardio isquémico con baja movilización y direccionamiento hacia este tejido de las células madre trasplantadas que permitan sus efectos auto y paracrinos; si se tiene en cuenta que estos procesos son dependientes de demandas, que el principal estímulo es la hipoxia y que esta se puede inducir en forma controlada mediante una prueba de esfuerzo, puede considerarse un pre-condicionamiento isquémico con esta metodología como método de señalización biomolecular. En este posible mecanismo median varios factores $(38,41,42)$, que inician 2 grandes procesos inducidos por la isquemia miocárdica: $1^{\circ}$ liberación de señales químicas, especialmente factor de crecimiento endotelial vascular (VEGF) que es el factor angiogénico mas importante, y citoquinas(CQ) como el factor estimulante de colonias de granulocitos (G-CSF), el factor de movilización y quimioquinas (QQ) SDF1, factor quimiotáctico, y este es uno de los efectos más importantes inducidos por el pre-condicionamiento isquémico ya que se pretende especialmente atracción y direccionamiento de las células madre hacia el miocardio hipoperfundido donde está el problema. $2^{\circ}$ Expresión de receptores: $\mathrm{El}$ SDF1 funciona como un ligando y requiere un receptor de anidación CXCR4 para crear el eje SDF1-CXCR 4, que junto con las otras señales químicas inicia la cascada de efectos directos y especialmente indirectos que inducen neo-vascularización.

Por las consideraciones anteriores, el trasplante autólogo "potenciado" de células madre CD34+ de médula ósea, puede ser una posibilidad terapéutica complementaria en pacientes con angina refractaria, en términos de mejoría de angina y calidad de vida.

Tabla 1. Criterios de inclusión y exclusión

\begin{tabular}{|c|c|}
\hline & CRITERIO \\
\hline INCLUSIÓN & $\begin{array}{l}\text { - Diagnóstico angiográfico de enfermedad coronaria significativa diferente } \\
\text { - } \text { D tronco no susceptible de revascularización coronaria percutánea ni quirúrgica. } \\
\text { - } \quad \text { Documento de isquemia por ecocardiograma ejercicio. } \\
\text { - Cualquier fracción de eyección. } \\
\text { - Edad: } 20-80 \text { años } \\
\text { - Cualquier sexo }\end{array}$ \\
\hline EXCLUSIÓN & $\begin{array}{l}\text { - Síndrome coronario agudo menor de } 3 \text { meses } \\
\text { - } \text { Otras causas de angina diferentes a enfermedad coronaria } \\
\text { - Embarazo o probabilidad del mismo } \\
\text { - Enfermedad cardiaca valvular, miocárdica no isquémica o pericárdica significativa o activa. } \\
\text { - Infermedades terminales o comorbilidad importante } \\
\text { Ind para realizar prueba de esfuerzo protocolo de Bruce modificado. C }\end{array}$ \\
\hline
\end{tabular}




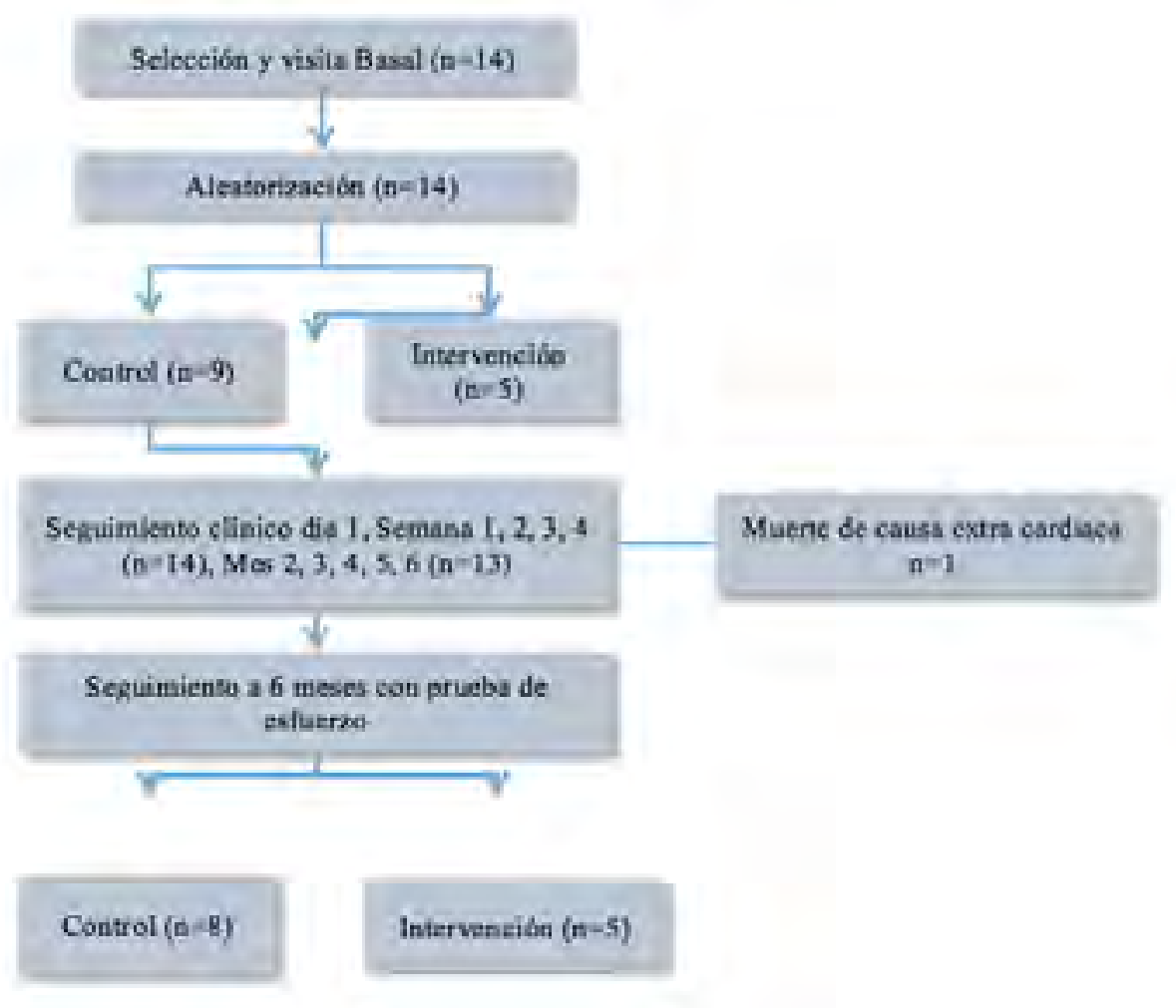

Figura 1. Estudio, elegibilidad, Aleatorización y seguimiento

\section{Materiales y Métodos}

Después de recibir la aprobación del Comité de Ética (Código 2011-07) se realizó un estudio piloto con 14 pacientes con angina refractaria, clase funcional clínica mayor o igual a III (NYHA y CCS), del Servicio de Cardiología del Hospital Militar Central, los pacientes cumplieron los criterios de inclusión incluidos en la Tabla 1. y después de firmar el consentimiento informado fueron asignados aleatoriamente a 2 grupos (Figura 1), el primero (intervención), con trasplante autólogo potenciado de células madre $\mathrm{CD} 34^{+}$de médula ósea por vía intra venosa más tratamiento médico convencional, en total 5 pacientes y el segundo con tratamiento médico convencional en total 9 pacientes. El desenlace principal se evaluó en términos de cambio en el umbral de isquemia/angina en mets y clase funcional a los seis meses respecto a las determinaciones basales, mediante prueba de esfuerzo protocolo de Bruce modificado con el fin de determinar los cambios en los dos grupos (Intervención versus control).

El diagnóstico de angina refractaria se hizo por historia clínica con verificación de óptimo tratamiento conven- cional posible de acuerdo a las guías vigentes $(5,44)$, la clase funcional clínica de ingreso fue definida de acuerdo a la clasificación NYHA $(45)$ y CCS $(46,47)$, y el documento de isquemia se estableció mediante ecocardiograma estrés ejercicio(48), con prueba de esfuerzo protocolo Bruce modificado $(49,50)$, en el que se definió adicionalmente el umbral de angina en mets, medida utilizada como unidad de consumo energético, indicadora de actividad aeróbica de acuerdo a la carga y al tiempo de ejercicio, y su correspondencia con la determinación ergométrica de la clase funcional.

El diagnóstico de la anatomía coronaria se hizo mediante arteriografía y la decisión de no opción de revascularización y aprobación para trasplante de células madre se realizó en junta de cardiología del Hospital Militar Central. En el grupo de intervención, el precondicionamiento isquémico se hizo mediante prueba de esfuerzo protocolo Bruce modificado $(18,19)$, hasta alcanzar el nivel de isquemia. Las células madre CD34+ fueron infundidas 10 horas después, tiempo óptimo de movilización y activación de $\mathrm{CD}^{2} 4^{+}(51)$. 


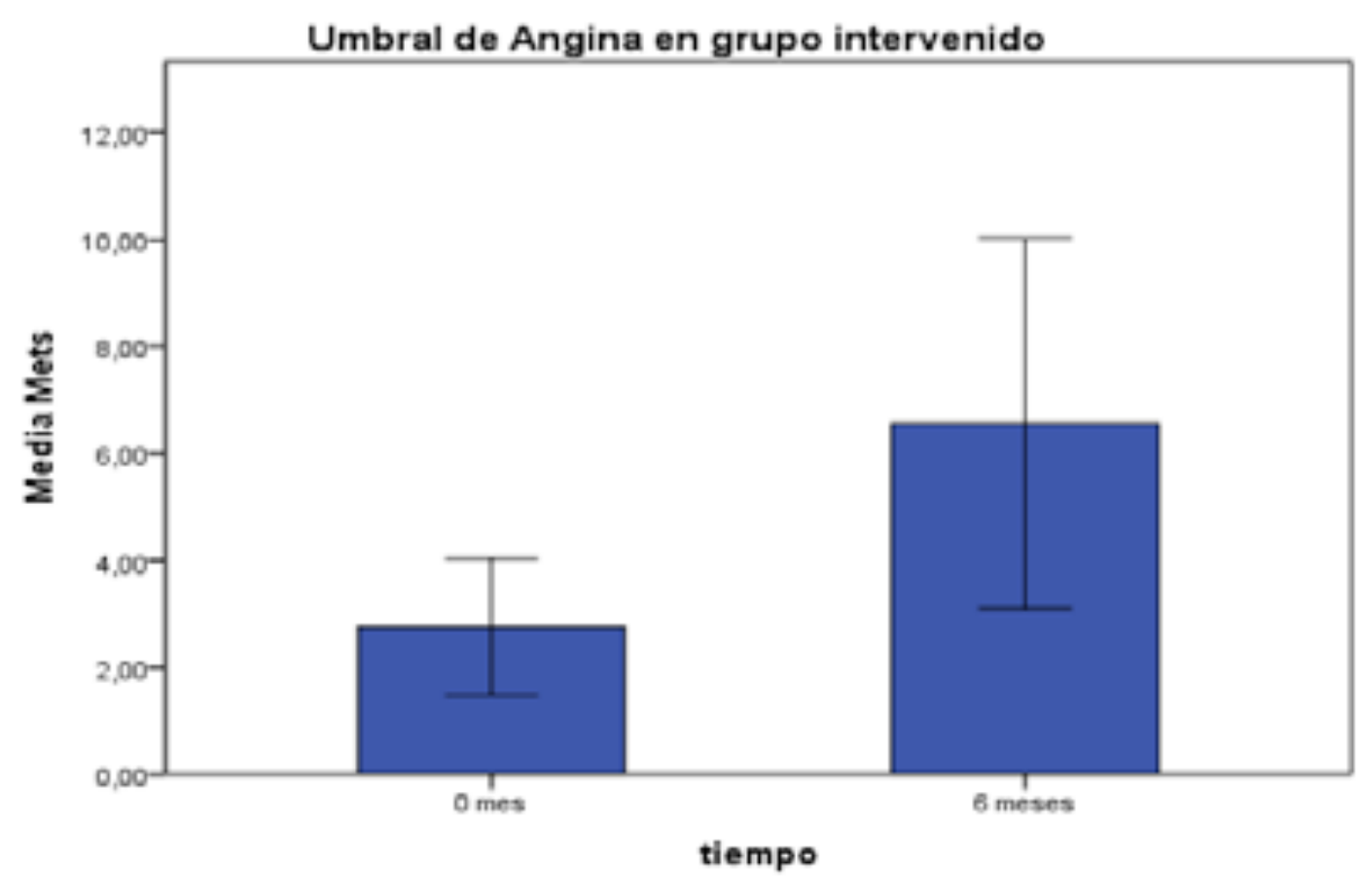

Barras de error: $95 \%$ IC

Figura 2. Umbral de angina en grupo de intervención. Cambio en el umbral de angina en mets de 0 mes (Basal) a 6 meses

La obtención de la médula ósea se realizó por la técnica de punción ósea con aguja en la cresta ilíaca posterior $(52,53)$, obteniendo un volumen de $100 \mathrm{~mL}$. Las Células mononucleares fueron obtenidas mediante gradiente de densidad en sala blanca en condiciones de esterilidad (54), una vez obtenidas se determinó el número de células mononucleares y células madre CD34+. Se realizó marcación con anticuerpos monoclonales para $\mathrm{CD}^{2} 5^{+} / \mathrm{CD}_{34}{ }^{+}(55)$ (según protocolo ISHAGE para células madre hematopoyéticas), $\mathrm{CD}_{133^{+}}$los cuales fueron analizadas en los programas Facs Canto y Facs Diva en el citómetro de flujo Facs Canto II ${ }^{\circledR}$ de BD y VEGFR2 mediante ELISA. El conteo de células madre se basó en el número de $\mathrm{CD} 4^{+}$y se estableció viabilidad con 7AAD. Para la administración de las células fueron enviadas en $50 \mathrm{~mL}$ de solución salina con $1 \%$ de albumina sérica humana. El trasplante de células madre se realizó por vena cefálica, basílica o ante cubital en un lapso de 15-20 minutos. El seguimiento durante las primeras 24 horas incluyó control clínico y electrocardiograma al ingreso y al egreso, posteriormente se realizó control clínico cada semana por 1 mes y se continuó cada mes hasta completar 6 meses.
Durante el seguimiento el tratamiento fue similar para el grupo de intervención y el grupo control, incluyó tratamiento convencional (6), y programa de rehabilitación cardiaca $(56,57)$, el análisis estadístico del desenlace primario se hizo contrastando las medianas de cambio en el valor del umbral de clase funcional para los dos grupos como muestras independientes con la prueba no paramétrica de Wilcoxon-Mann-Whitney, los datos fueron procesados mediante paquete estadístico STATA 12.

\section{Resultados}

Se realizó seguimiento a todos los pacientes del grupo intervención y 8 pacientes del grupo control, ya que un paciente falleció por causa extra cardiaca.

No hubo diferencias significativas en las características basales entre el grupo con intervención y el grupo control en ninguno de los parámetros analizados (Tabla 2). El número de $\mathrm{CD} 34^{+}$trasplantado promedio fue de $16.256 \times 10 \otimes$ (SD $7.522 \times 10$ × ) y viabilidad de $91.93 \%$ (SD 4.81). En el grupo de pacientes con inter- 
Tabla 2. Caracteristicas de la población al 0 mes antes de la intervención.

\begin{tabular}{|c|c|c|c|}
\hline Variable & Grupo de intervención n:5 & Grupo control n:9 & Valor $\mathbf{P}^{*}$ \\
\hline \multicolumn{4}{|l|}{ Edad (Años) } \\
\hline Mediana & 69 & 69 & 0.8 \\
\hline RIQ & $62-74$ & $65-72$ & \\
\hline \multicolumn{4}{|l|}{ Sexo } \\
\hline Masculino n (\%) & $2(40)$ & $4(4)$ & 0.7 \\
\hline Femenino n (\%) & $3(60)$ & $5(56)$ & \\
\hline \multicolumn{4}{|l|}{ IMC } \\
\hline Mediana & 24 & 27 & 0.31 \\
\hline RIQ & $23-28$ & $23.5-33$ & \\
\hline \multicolumn{4}{|l|}{ FE\% (VI) } \\
\hline Mediana & 58 & 50 & 0.94 \\
\hline RIQ & $61-63$ & $48-60$ & \\
\hline Número Vasos & & & 0.65 \\
\hline $1 \mathrm{n}(\%)$ & $1(20)$ & $3(33)$ & \\
\hline $2 \mathrm{n}(\%)$ & $3(80)$ & $5(55)$ & \\
\hline 3 n (\%) & $1(20)$ & $1(11)$ & \\
\hline \multicolumn{4}{|c|}{ Umbral angina/isquemia (Mets) } \\
\hline Mediana & 3.3 & 3.7 & 0.18 \\
\hline RIQ & $3.2-3.5$ & $3.4-5$ & \\
\hline \multicolumn{4}{|l|}{ Clase Funcional (PE.B.M) } \\
\hline III & 4 & 4 & \\
\hline IIc & 1 & 2 & \\
\hline IIb & 0 & 3 & \\
\hline \multicolumn{4}{|c|}{ STRAIN longitudinal \% (VI) } \\
\hline Mediana & 14 & 16 & 0.94 \\
\hline RIQ & $20-14$ & $18.5-14.5$ & \\
\hline \multicolumn{4}{|l|}{ Factores de Riesgo n (\%) } \\
\hline HTA & $3(60)$ & $7(78)$ & 0.48 \\
\hline $\mathrm{DM}$ & $1(20)$ & $2(22)$ & 0.92 \\
\hline DLP & $4(80)$ & $9(100)$ & 0.16 \\
\hline Tabaquismo & 0 & 0 & \\
\hline
\end{tabular}




\begin{tabular}{lccc}
\hline \multicolumn{1}{c}{ Variable } & Grupo de intervención $\mathbf{n : 5}$ & Grupo control n:9 & Valor P* \\
\hline Intervenciones Cardiacas $\mathbf{n}(\%)$ & & & \\
\hline RVC CX & $1(22)$ & $2(20) 0.92$ & 0.48 \\
\hline PCI & $2(40)$ & $3(22)$ & \\
\hline Fármacos $\quad \mathbf{n}(\%)$ & & & \\
\hline Antiagregantes plaquetarios & $5(100)$ & $9(100)$ & 0.15 \\
\hline Beta bloqueadores & $5(100)$ & $9(100)$ & 0.65 \\
\hline Anti cálcicos & $5(100)$ & $6(67)$ & $8(88)$ \\
\hline Nitratos & $4(80)$ & $9(100)$ & $7(77)$ \\
\hline Estatinas & $5(100)$ & $3(60)$ & 0.48 \\
\hline I-ECA/ARAII & & & \\
\hline
\end{tabular}

* Nivel de significancia mediante la prueba no paramétrica de Wilcoxon-Mann-Whitney, IMC: Índice de masa corporal, FE(VI): Fracción de eyección del ventrículo izquierdo, Mets: unidad de consumo metabólico (1 Mets = 3,5 ccO $/ 2 \mathrm{~kg} / \mathrm{min}$.), STRAIN(VI): Deformación Longitudinal del ventrículo izquierdo. HTA: Hipertensión arterial, DM: Diabetes mellitus, DLP: Dislipidemia, RVC: revascularización coronaria quirúrgica, PCI: intervención coronaria percutánea. I-ECA: Inhibidores de enzima convertidora de angiotensina, ARA-II: Inhibidores de receptores de angiotensina II, Clase funcional (PE.BM): clase funcional medida en prueba de esfuerzo con protocolo Bruce modificado.

vención se encontró aumento significativo en el umbral de angina/isquemia (Tabla 3, Figura 2) con mejoría en la tolerancia al ejercicio, y clase funcional (Tabla 4), no hubo mejoría en un paciente de este grupo y no se encontraron predictores de respuesta. En el grupo control no hubo cambios significativos (Tabla 3). No hubo complicaciones inherentes al procedimiento.

\section{Discusión}

En este estudio piloto los resultados permiten considerar que el trasplante autólogo de células madre $\mathrm{CD} 34^{+}$de médula ósea, potenciado mediante pre-condicionamiento isquémico, con un método de baja complejidad y bajo riesgo, es potencialmente benéfico para los pacientes con angina refractaria, hasta el momento considerados "pacientes sin opción". Aunque la muestra es pequeña , se puede plantear teóricamente que el pre-condicionamiento isquémico puede favorecer la potenciación de los efectos del trasplante de este tipo de células madre, efecto que estaría, en primer lugar, dado por activación de los procesos de quimiotaxis, movilización, direccionamiento y anidación de las células madre trasplantadas hacia el miocardio isquémico, con inicio de sus efectos autocrinos, donde las células que logran anidarse y diferenciarse pueden tener un efecto directo autocrino bajo; en segundo lugar, por activación de los mecanismos paracrinos de las células madre trasplantadas con un efecto indirecto mayor, posiblemente responsable de gran parte de la neovacularizacion y sus beneficios; el mecanismo que favorece que el efecto se centre en el miocardio isquémico está mediado por señalización bio molecular, aunque como ha sido analizado por otros autores (14), la densidad celular óptima requerida para promover estos efectos no está aún definida; estas consideraciones se apoyan en los estudios de análisis general de trasplante de células madre en enfermedades cardiacas, donde se ha encontrado que la retención y la diferenciación de células madre es muy baja con cualquier metodología (36-39) y a pesar de esto, los resultados son positivos en términos de bio actividad cardiovascular (15).

Si se considera que la baja retención y diferenciación de células madre no puede explicar los beneficios solo por efectos directos, hay que plantear que el efecto indirecto paracrino sea muy importante, probablemente más que el efecto directo (40). El tiempo del trasplante de células madre después de inducir la isquemia se estableció buscando administrarlas en el momento óptimo de activación de factores de movilización y estimulación de $\mathrm{CD} 4^{+}$tratando de optimizar los mecanismos autocrinos y paracrinos de las células madre 
Tabla 3. Cambio en el umbral de angina en los pacientes intervenidos vs. control.

\begin{tabular}{|c|c|c|c|c|c|c|}
\hline \multirow{2}{*}{ GRUPO } & \multirow{2}{*}{ PACIENTE } & \multicolumn{4}{|c|}{ UMBRAL DE ANGINA (METS) } & \multirow[b]{2}{*}{ Valor $\mathbf{P}^{* *}$} \\
\hline & & Omes & 6 meses & Cambio & \% cambio & \\
\hline \multirow[t]{2}{*}{ INTERVENCIÓN } & $\begin{array}{l}1 \\
2 \\
3 \\
4 \\
5\end{array}$ & \begin{tabular}{|l}
4,6 \\
3,3 \\
3,5 \\
3,2 \\
3,2
\end{tabular} & \begin{tabular}{|l|}
10,0 \\
7,8 \\
7,0 \\
5,5 \\
2,5
\end{tabular} & $\begin{array}{l}5,4 \\
4,5 \\
3,5 \\
2,3 \\
-0,7\end{array}$ & \begin{tabular}{|l}
117 \\
136 \\
100 \\
72 \\
-22
\end{tabular} & 0.02 \\
\hline & $\mathrm{X}(\mathrm{DS})$ & $3,6(0.6)$ & $6,6(2.8)$ & $3,0(2.2)$ & $84(55)$ & \\
\hline \multirow[t]{2}{*}{ CONTROL } & $\begin{array}{l}1 \\
2 \\
3 \\
4 \\
5 \\
6 \\
7 \\
8 \\
9\end{array}$ & \begin{tabular}{|l}
3,2 \\
5,0 \\
5,5 \\
2,5 \\
5,2 \\
3,5 \\
4,4 \\
3,7 \\
4,9
\end{tabular} & \begin{tabular}{|l}
3,5 \\
4,0 \\
$*$ \\
3,0 \\
4,2 \\
3,0 \\
3,0 \\
2,5 \\
4,0
\end{tabular} & \begin{tabular}{|l}
0,3 \\
$-1,0$ \\
$*$ \\
0,5 \\
$-1,0$ \\
$-0,5$ \\
$-1,4$ \\
$-1,2$ \\
$-0,9$
\end{tabular} & \begin{tabular}{|l}
9 \\
-20 \\
$*$ \\
20 \\
-19 \\
-14 \\
-32 \\
-32 \\
-18
\end{tabular} & $>0.05$ \\
\hline & $\mathrm{X}(\mathrm{DS})$ & $4,2(1.0)$ & $3,28(0.66)$ & $-0,9(0.7)$ & -22 & \\
\hline
\end{tabular}

Met: unidad de consumo metabólico $\left(1 \mathrm{Met}=3,5 \mathrm{ccO}_{2} / \mathrm{kg} / \mathrm{min}\right.$. ) *Muerte por causa extra cardiaca, ${ }^{* *}$ nivel de significancia mediante la prueba no paramétrica de Wilcoxon-Mann-Whitney.

trasplantadas ya mencionados (y no para activar los mecanismos de compensación primarios ya agotados en estos pacientes). Teniendo en cuenta estas consideraciones, especialmente la quimiotaxis, la importancia del flujo heterocoronario, además del homocoronario hacia la microcirculación del miocardio isquémico, el tamaño de las células madre $\mathrm{CD}^{3} 4^{+}$y su tránsito (35), se escogió la vía de administración intravenosa como una opción útil, segura y simple, sin desconocer la importancia de las otras vías y el potencial beneficio del pre condicionamiento isquémico con otros métodos de administración. A pesar de los obstáculos mencionados y de la falta de evidencia del mecanismo exacto de la administración sistémica, ayudan a soportar la utilización de esta vía, los estudios experimentales en modelos en ratones y porcinos de isquemia, con ad- ministración de células madre por vía intravenosa con evidencia de angiogénesis por inmuno histoquímica, aumento de perfusión miocárdica, aumento de densidad capilar $(58,59)$, y los reportes clínicos promisorios (60); además la consideración de que uno de los determinantes de la utilidad de esta vía en post infarto miocárdico es la señalización miocárdica dada por la injuria (61), concepto importante en nuestro estudio donde la señalización mediante pre-condicionamiento isquémico es un aspecto básico.

No se puede hacer un análisis comparativo de nuestros resultados con otros previamente publicados, ya que no hay estudios con metodología similar, sin embargo, el hallazgo de mejoría en términos tolerancia al ejercicio y sus equivalentes es consistente con algunos publicados en 
estudios con métodos diferentes de mayor complejidad que incluyen administración intramiocárdica y/o GCSF entre otros $(14,22,24,25)$. El tamaño de la muestra no tiene el poder suficiente para obtener conclusiones definitivas puesto que las diferencias encontradas pudiesen ser debidas a un error tipo I, pero sí permite inferir la importancia de trabajar no solamente sobre las condiciones de las células madre trasplantadas, también sobre el nicho receptor y factores adicionales que puedan potenciar sus beneficios, en un contexto global de medicina regenerativa cardiovascular y continuar buscando opciones para mejorar la calidad de vida de estos pacientes.

En resumen, en esta investigación piloto el grupo de pacientes con angina refractaria intervenidos con trasplan- te autólogo de células madre de médula ósea CD34+ por vía intravenosa, potenciado mediante pre condicionamiento isquémico, se asoció a mejoría del umbral de isquemia/angina y clase funcional a 6 meses. No hubo complicaciones durante el procedimiento. Por la limitación del tamaño de la muestra se requiere un estudio con poder suficiente para validar estos resultados y establecer predictores de respuesta.

\section{Conflicto de Intereses}

Los autores declaran no tener de manera directa o indirecta, algún tipo de conflicto de intereses financieros, académicos o laborales que puedan poner en peligro la validez de este estudio.

\begin{tabular}{|l|l|l|l|}
\hline \multirow{2}{*}{ GrupoGrupo } & Paciente & \multicolumn{2}{|c|}{ Clase Funcional * } \\
& & mes 6 meses \\
\hline Intervención & 1 & IIC & IB \\
& 2 & III & IC \\
& 3 & III & IC \\
& 4 & III & IB \\
& 5 & III & III \\
\hline Control & 1 & III & III \\
& 2 & IIB & IIC \\
& 3 & IIB & \\
& 4 & III & III \\
& 5 & IIB & IIC \\
& 6 & III & III \\
& 7 & IIC & III \\
& 8 & III & III \\
& 9 & IIC & IIC \\
\hline
\end{tabular}

Tabla 4. Cambio de clase funcional en los pacientes intervenidos us control. *Clase funcional medida con prueba de esfuerzo 
Referencias

1. Bernstein SJ, Brorsson B, Aberg T, Emanuelsson H, Brook RH, Werkö L. Appropriateness of referral of coronary angiography patients in Sweden. SECOR/SBU Project Group. Heart. 1999; 81:470-477.

2. Povsic TJ, Broderick S, Anstrom KJ, Shaw LK, Ohman EM, Eisenstein EL, et al. Predictors of long-term clinical endpoints in patients with refractory angina. J Am Heart Assoc. 2015; 4:01287.

3. Hemingway H, Crook AM, Feder G, Banerjee S, Dawson JR, Magee $P$, et al. Underuse of coronary revascularization procedures in patients considered appropriate candidates for revascularization. N Engl J Med. 2001; 344:645-54.

4. Serruys PW, Andrew T.L, Lex A. Herwerden, J. Sousa E, Jatene A, et al. Five-Year Outcomes After Coronary Stenting Versus Bypass Surgery for the Treatment of Multivessel Disease. The Final Analysis of the Arterial Revascularization Therapies Study (ARTS) Randomized Trial, J Am Coll Cardiol. 2005; 46(4):575-581.

5. Fihn SD, Gardin JM, Abrams J, Berra K, Blankenship JC, Dallas AP, et al. ACCF/AHA/ACP/AATS/PCNA/SCAI/ STS Guideline for the Diagnosis and Management of $\mathrm{Pa}$ tients With Stable Ischemic Heart Disease: A Report of the American College of Cardiology Foundation/American Heart Association Task Force on Practice Guidelines, and the American College of Physicians, American Association for Thoracic Surgery, Preventive Cardiovascular Nurses Association, Society for Cardiovascular Angiography and Interventions, and Society of Thoracic Surgeons. Journal of the American College of Cardiology. 2012; 60(24): e44-e164.

6. Montalescot G, Sechtem U, Achenbach S, Andreotti F, Arden C, Budaj A, et al. Guía de práctica clínica de la ESC 2013 sobre diagnóstico y tratamiento de la cardiopatía isquémica estable. Rev Esp. Cardiol. 2014; 67(2): 135. e1-e81.

7. Bernstein SJ, Brorsson B, Aberg T, Emanuelsson H, Brook RH, Werkö L. Appropriateness of referral of coronary angiography patients in Sweden. SECOR/SBU Project Group. Heart. 1999; 81:470-477.

8. Mukherjee D, Bhatt DL, Roe MT, Patel V, Ellis SG. Direct myocardial revascularization and angiogenesis-how many patients might be eligible. Am J Cardiol. 1999; 84:598600.

9. Henry TD, Satran D, Hodges JS, Johnson RK, Poulose AK, Campbell AR, et al. Long-term survival in patients with refractory angina. Eur Heart J. 2013; 34:2683-2688.

10. Mukherjee D, Comella K, Bhatt DL, Roe MT, Patel V, Ellis SG. Clinical outcome of a cohort of patients eligible for therapeutic angiogenesis or transmyocardial revasculariza- tion. Am Heart J. 2001; 142:72-74.

11. Kandzari DE, Lam LC, Eisenstein EL, Clapp-hanning N, Fine JT, Califf RM, et al. Advanced coronary artery disease: appropriate end points for trials of novel therapies. Am Heart J. 2001; 142:843-851.

12. Loh PH, Cleland JGF, Louis AA, Kennard ED, Cook JF, Caplin JL, et al. Enhanced external counterpulsation in the treatment of chronic refractory angina: a long-term follow-up outcome from the international enhanced external counterpulsation patient registry. Clin Cardiol. 2008; 31:159-164.

13. Mohr FW, Morice MC, Kappetein AP, Feldman TE, Ståhle $\mathrm{E}$, Colombo A, et al. Coronary artery bypass graft surgery versus percutaneous coronary intervention in patients with three-vessel disease and left main coronary disease: 5-year follow-up of the randomised, clinical SYNTAX trial. Lancet. 2013; 381:629-638.

14. Losordo DW, Henry TD, Davidson C, Sup Lee J, Costa MA, Bass T, et al. ACT34-CMI Investigators. Intramyocardial, autologous CD34+ cell therapy for refractory angina. Circ Res. 2011; 5; 109(4):428-36.

15. Asahara T, Murohara T, Sullivan A, Silver M, van der Zee $\mathrm{R}, \mathrm{Li} \mathrm{T}$, et al. Isolation of putative progenitor endothelial cells for angiogenesis. Science. 1997; 275:964-967.

16. Menasché P, Hagege AA, Scorsin M, Pouzet B, Desnos M, Dubo D, et al: Myoblast transplantation for heart failure. Lancet. 2001, 357: 279 - 280.

17. Kawamoto A, Iwasaki H, Kusano K, Murayama T, Oyamada A, Silver M, et al. Cd34-positive cells exhibit increased potency and safety for therapeutic neovascularization after myocardial infarction compared with total mononuclear cells. Circulation. 2006; 114:2163-2169.

18. Iwasaki H, Kawamoto A, Ishikawa M, Oyamada A, Nakamori S, Nishimura $\mathrm{H}$, et al. Dose-dependent contribution of cd34-positive cell transplantation to concurrent vasculogenesis and cardiomyogenesis for functional regenerative recovery after myocardial infarction. Circulation. 2006; 113:1311-1325.

19. Zeng L, Hu Q, Wang X, Mansoor A, Lee J, Feygin J, et al, Bioenergetic and Functional Consequences of Bone Marrow-Derived Multipotent Progenitor Cell Transplantation in Hearts With Postinfarction Left Ventricular Remodeling, Circulation. 2007; 115: 1866-1875.

20. Pokushalov E, Romanov A, Chernyavsky A, Larionov P, Terekhov I, Artyomenko S, et al. Efficiency of intramyocardial injections of autologous bone marrow mononuclear cells in patients with ischemic heart failure: a randomized study. J Cardio vasc Transl Res. 2010; 3: 160-168.

21. Perin EC, Silva GV, Henry TD, Cabreira-Hansen MG, Moore WH, Coulter SA, et al. A randomized study of transendocardial injection of autologous bone marrow mononu- 
clear cells and cell function analysis in ischemic heart failure (FOCUS-HF). Am Heart J. 2011; 161: 1078-1087.

22. Van Ramshorst J, Bax JJ, Beeres SL, Dibbets-Schneider P, Roes SD, Stokkel MP, et al. Intramyocardial bone marrow cell injection for chronic myocardial ischemia: a randomized controlled trial. JAMA. 2009; 301: 1997-2004.

23. Wang S, Cui J, Peng W, Lu M: Intracoronary autologous CD34+ stem cell therapy for intractable angina. Cardiology. 2010; 117: 140-147.

24. Losordo DW, Schatz RA, White CJ, Udelson JE, Veereshwarayya $\mathrm{V}$, Durgin $\mathrm{M}$, et al. Intramyocardial transplantation of autologous cd34_ stem cells for intractable angina: a phase i/iia double-blind, randomized controlled trial. Circulation. 2007; 115:3165-3172.

25. Kovacic JC1, Macdonald P, Feneley MP, Muller DW, Freund J, Dodds A, et al. Safety and efficacy of consecutive cycles of granulocyte-colony stimulating factor, and an intracoronary CD133+cell infusion in patients with chronic refractory ischemic heart disease: The G-CSF in Angina patients with IHD to stimulate Neovascularization (GAIN I) trial, AHJ November 2008. Volume 156, Issue 5, Pages 954-963

26. Tse HF, Thambar S, Kwong YL, Rowlings P, Bellamy G, McCrohon $\mathrm{J}$, et al. Prospective randomized trial of direct endomyocardial implantation of bone marrow cells for treatment of severe coronary artery diseases (PROTECT-CAD trial). Eur Heart J 2007, 28:2998-3005.

27. Li N, Yang YJ, Zhang Q, Jin C, Wang H, Qian HY, et al. Stem cell therapy is a promising tool for refractory angina: a meta-analysis of randomized controlled trials. Can J Cardiol. 2013; 29:908-914.

28. Fisher SA, Dorée C, Brunskill SJ, Mathur A, Martin-Rendon $\mathrm{E}$. Bone marrow stem cell treatment for ischemic heart disease in patients with no option of revascularization: a systematic review and meta-analysis. PLoS One. 2013; 8: e64669.

29. Povsic J, Junge C, Nada A, Schatz RA, Harrington RA, Davidson CJ, et al. RENEW. A phase 3, randomized, double-blinded, active-controlled, unblinded standard of care study assessing the efficacy and safety of intramyocardial autologous CD34+ cell administration in patients with refractory angina: Design of the RENEW study Thomas, Am Heart J. 2013;165:854-861.

30. Jiménez-Quevedo P, González-Ferrer J, Sabate M, GarcíaMoll X, Delgado-Bolton R, Llorente L, et al. Promote Angiogenesis in Patients with Refractory Angina Final Results of the PROGENITOR Randomized TrialCirc Res. 2014; 115:950-960.

31. Iwasaki H1, Kawamoto A, Ishikawa M, Oyamada A, Nakamori S, Nishimura H Et al. Dose-Dependent Contribution of CD34-Positive Cell Transplantation to Concurrent Vas- culogenesis and Cardiomyogenesis for Functional Regenerative Recovery After Myocardial Infarction, Circulation. 2006; 113: 1311-1325

32. Leri A, Anversa P. Stem Cells and Myocardial Regeneration Cooperation Wins Over Competition, Circulation. 2013; 127: 165-168.

33. Freyman T1, Polin G, Osman H, Crary J, Lu M, Cheng $\mathrm{L}$, et al, A quantitative, randomized study evaluating three methods of mesenchymal stem cell delivery following myocardial infarction, European Heart Journal. 2006; 27(9): $1114-1122$.

34. Fischer UM1, Harting MT, Jimenez F, Monzon-Posadas WO, Xue H, Savitz SI, et al. Pulmonary Passage is a Major Obstacle for Intravenous Stem Cell Delivery: The Pulmonary First-Pass Effect, Stem Cells Dev. 2009 Jun; 18(5): 683-691. Stem Cells Dev. 2009; 18(5): 683-691.

35. Hou D1, Youssef EA, Brinton TJ, Zhang P, Rogers P, Price ET, et al. Radiolabeled Cell Distribution after Intramyocardial, Intracoronary, and Retrograde Venous Delivery: CMN, Circulation. 2005; 112(Suppl I): I150-I156.

36. Aicher A1, Brenner W, Zuhayra M, Badorff C, Massoudi $\mathrm{S}$, Assmus B, et al. Assessment of the tissue distribution of transplanted human endothelial progenitor cells by radioactive labeling. Circulation. 2003; 107:2134-9.

37. Blocklet D1, Toungouz M, Berkenboom G, Lambermont M, Unger P, Preumont N, et al. Myocardial homing of nonmobilized peripheral-blood CD34+ Cells after intracoronary injection. Stem Cells. 2006; 24:333-6.

38. Hofmann M, Wollert KC, Meyer GP, et al. Monitoring of Bone Marrow Cell Homing into the Infarcted Human Myocardium. Circulation. 2005; 111:2198-2202.

39. Malliaras K, Zhang Y, Seinfeld J, Galang G, Tseliou E, Cheng $\mathrm{K}$, et al. Cardiomyocyte proliferation and progenitor cell recruitment underlie therapeutic regeneration after myocardial infarction in the adult mouse heart. EMBO Mol Med. 2013; 5: 191 - 209.

40. Ghadge SK, Muhlstedt S, Ozcelik C, Bader M. SDF-1alpha as a therapeutic stem cell homing factor in myocardial infarction. Pharmacol Therapeut. 2011; 129:97-108.

41. Strauer BE1, Brehm M, Schannwell CM. The therapeutic potential of stem cells in heart disease, Cell Prolif. 2008; 41 (Suppl. 1), 126-145.

42. Adams V1, Lenk K, Linke A, Lenz D, Erbs S, Sandri M, et al. Increase of Circulating Endothelial Progenitor Cells in Patients with Coronary Artery Disease After Exercise-Induced Ischemia. Arteriosclerosis, Thrombosis, and Vascular Biology. 2004; 24: 684-90.

43. World Medical Association. World Medical Association Declaration of Helsinki Ethical Principles for Medical Research Involving Human Subjects. JAMA. 2013; 310(20): 2191-2194. 
44. Mannheimer C, Camici P, Chester MR, Collins A, DeJongste $\mathrm{M}$, Eliasson $\mathrm{T}$, et al. The problem of chronic refractory angina; report from the ESC Joint Study Group on the Treatment of Refractory Angina. Eur Heart J. 2002; 23:355-70.

45. .The Criteria Committee of the New York Heart Association. Nomenclature and Criteria for Diagnosis of Diseases of the Heart and Great Vessels. 9th ed. Boston, Mass: Little, Brown \& Co. 1994; 253-256.

46. Campeau L: Canadian Cardiovascular Society Functional classification of angina pectoris [letter]. Circulation. 1976; 54:522.

47. Kavita Sharma, Priya Kohli, Martha Gulati, FACC, FAHA, An Update on Exercise Stress Testing, Curr Probl Cardiol. 2012; 37:177-202.

48. Pellikka PA1, Nagueh SF, Elhendy AA, Kuehl CA, Sawada SG; American Society of Echocardiography, American Society of Echocardiography Recommendations for Performance, Interpretation, and Application of Stress Echocardiography. Journal of the American Society. 2007; 20(9): 1021-1041.

49. Gibbons RJ, Balady GJ, Bricker JT, Chaitman BR, Fletcher GF, Froelicher VF, et al. American College of Cardiology/American Heart Association Task Force on Practice Guidelines. Committee to Update the 1997 Exercise Testing Guidelines. ACC/AHA 2002 guideline update for exercise testing: summary article. A report of the American College of Cardiology/American Heart Association Task Force on Practice Guidelines (Committee to Update the 1997 Exercise Testing Guidelines). J Am Coll Cardiol. $2002 ; 16 ; 40(8): 1531-40$.

50. Fletcher GF, Balady GJ, Amsterdam EA, Chaitman B, Eckel R, Fleg J, et al. Exercise Standards for Testing and Training A Statement for Healthcare Professionals from the American Heart Association. Circulation. 2001; 104:1694-1740.

51. Blanco-Guarín MI, Avila-Portillo LM, Arango A, Avila J, Vásquez JP, Álvarez O, et al. Medición en diferentes periodos de tiempo de factores asociados a anidación de células madre, en pacientes con falla cardiaca y angina refractaria, posterior a estimulación cardiaca controlada. Revista Med. 2013;21 (suplemento 1): S22.

52. Demko SG. Transplantation procedures. En: Burt RK, Deeg HJ, Santos GW. On call in Bone Marrow Transplantation.Chapman and Hall, New York, 1996; pág.: 39-51.
53. Confer DL, Stroncek DF. Bone Marrow and peripheral blood stem cell donors. En: Thomas ED, Blume KG, Forman SJ (eds.). Hematopoietic cell transplantation. Blackwell Scientific Inc. Malden, 1999;421-30.

54. Hibino N, Nalbandian A, Devine L, Martinez RS, et al. Comparison of human bone marrow mononuclear cell isolation methods for creating tissue-engineered vascular grafts: novel filter system versus traditional density centrifugation method. Tissue Engineering Part C Methods. 2011;17(10):993-8

55. Pranke P., Hendrikx J., Alespeiti4 G., Nardi N., Rubinstein P. Et al. Comparative quantification of umbilical cord blood CD34+ and CD34+ bright cells using the ProCount ${ }^{\mathrm{TM}}-\mathrm{BD}$ and ISHAGE protocols. Brazilian Journal of medical and Biological Research. 2006; 39: 901-906

56. AHA/AACVPR Scientific Statement. Core Components of Cardiac Rehabilitation/Secondary Prevention Programs 2007 Update. Balady G. Et al. Circulation. 2007; 115:2675-2682.

57. American Association of Cardiovascular and Pulmonary Rehabilitation: Guidelines for Cardiac Rehabilitation and Secondary Prevention Programs, Fourth Edition, Human Kinetics, 2004.

58. E. Halkos, Z. Q. Zhao, F. Kerendi et al., "Intravenous infusion of mesenchymal stem cells enhances regional perfusion and improves ventricular function in a porcine model of myocardial infarction," Basic Research in Cardiology, vol. 103, no. 6, pp. 525-536,2008.

59. Noritosi Nagaya, Takafumi Fujii, Takashi Iwase, Hajime Ohgushi, Takefumi Itoh et al, Intravenous administration of mesenchymal stem cells improves cardiac function in rats with acute myocardial infarction through angiogenesis and miogénesis, American Journal of Physiology - Heart and Circulatory Physiology, Published online before print July 29, 2004,1152.

60. J.M. Hare, J. H. Traverse, T. D. Henry et al., "A randomized, double-blind, placebo-controlled, dose-escalation study of intravenous adult human mesenchymal stem cells (prochymal) after acute myocardial infarction," Journal of the American College of Cardiology, vol. 54, no. 24, pp. 2277-2286, 2009.

61. Calvin C Sheng, Li Zhou, an Jijun Hao, Current Stem Delivery Methods for Myocardial repair, BioMed Research international vol 2013, Article ID547902, 1-15. 